\title{
6.1-MV, 0.79-MA laser-triggered gas switch for multimodule, multiterawatt pulsed-power accelerators
}

\author{
K. R. LeChien, ${ }^{1}$ W. A. Stygar, ${ }^{1}$ M. E. Savage, ${ }^{1}$ P. E. Wakeland ${ }^{2}$ V. Anaya, ${ }^{2}$ D. S. Artery, ${ }^{2}$ M. J. Baremore, ${ }^{2}$ D. E. Bliss, ${ }^{1}$ \\ R. Chavez, ${ }^{2}$ G. D. Coombs, ${ }^{2}$ J. P. Corley, ${ }^{2}$ P. A. Jones, ${ }^{2}$ A. K. Kipp, ${ }^{2}$ B. A. Lewis, ${ }^{2}$ J. A. Lott, ${ }^{2}$ J. J. Lynch, ${ }^{2}$ G. R. McKee, ${ }^{1}$ \\ S. D. Ploor, ${ }^{2}$ K. R. Prestwich, ${ }^{3}$ S. A. Roznowski, ${ }^{2}$ D. C. Spencer, ${ }^{2}$ S. D. White, ${ }^{2}$ and J. R. Woodworth ${ }^{1}$ \\ ${ }^{1}$ Sandia National Laboratories, Albuquerque, New Mexico 87185, USA \\ ${ }^{2}$ Ktech Corporation, Albuquerque, New Mexico 87123, USA \\ ${ }^{3}$ K.R. Prestwich Consulting, Albuquerque, New Mexico 87112, USA
}

(Received 29 October 2009; published 24 March 2010)

\begin{abstract}
A 6.1-MV, 0.79-MA laser-triggered gas switch (LTGS) is used to synchronize the 36 modules of the $Z$ machine at Sandia National Laboratories. Each module includes one switch, which serves as the last command-fired switch of the module, and hence is used to determine the time at which each module electrically closes relative to the other modules. The switch is $\sim 81-\mathrm{cm}$ in length, $\sim 45-\mathrm{cm}$ in diameter, and is immersed in mineral oil. The outer switch envelope consists of six corrugated monomer-cast acrylic insulators and five contoured stainless-steel rings. The trigger electrodes are fabricated from copperinfused tungsten. The switch is pressurized with several atmospheres of sulfur hexafluoride $\left(\mathrm{SF}_{6}\right)$, which is turbulently purged within 2 seconds after every shot. Each switch is powered from a 6-MV, 0.78-MJ Marx generator which pulse charges a $24-\mathrm{nF}$ intermediate-store water capacitor in $1.4-\mu \mathrm{s}$. Closure of the switch allows power to flow into pulse-forming transmission lines. The power pulse is subsequently compressed by water switches, which results in a total accelerator output power in excess of 70-TW. A previous version of the LTGS performed exceptionally at a 5.4-MV, 0.7-MA level on an engineering test module used for switch development. It exhibited a $1-\sigma$ jitter of $\sim 5 \mathrm{~ns}$, a prefire and flashover rate less than $0.1 \%$, and a lifetime in excess of 150 shots. When installed on the $Z$ accelerator, however, the switch exhibited a prefire probability of $\sim 3 \%$, a flashover probability of $\sim 7 \%$, and a 15 -ns jitter. The difference in performance is attributed to several factors such as higher total charge transfer, exposure to more debris, and more stressful dynamic mechanical loading upon machine discharge. Under these conditions, the replacement lifetime was less than ten shots. Since refurbishment of $Z$ in October 2007, there have been three LTGS design iterations to improve the performance at 6.1-MV. The most recent design exhibits a prefire rate of less than $0.1 \%$, a flashover rate of $\sim 0.2 \%$, a single switch jitter of $\sim 6$-ns, and a lifetime of greater than 75 shots. Modifications to achieve the performance improvement are detailed in this article.
\end{abstract}

DOI: 10.1103/PhysRevSTAB.13.030401

PACS numbers: 84.32.Dd

\section{INTRODUCTION}

Terawatt and petawatt class $z$-pinch accelerators require nanosecond synchronization of multiple mega-ampere pulsed-power drivers arranged in parallel [1]. The general architecture of these drivers consists of stages of energy storage and switching for spatial and temporal energy compression, delivering very high power densities to loads of interest. Depending on the type of experiment, the stored electrical energy is converted to magnetic pressure, mechanical energy, or radiated energy. Peak radiated power for a $z$-pinch wire array and peak magnetic pressure for an isentropic compression experiment scale with both current amplitude and pulse shape [2,3].

The typical architecture for one module of a megaampere driver consists of storing energy over several minutes in a Marx bank that is switched via relatively lowvoltage, low-current (200-kV, 200-kA) gas switches. The Marx bank charges intermediate storage capacitance on a microsecond time scale. Energy is subsequently switched via a multimegavolt, mega-ampere sulfur hexafluoride $\left(\mathrm{SF}_{6}\right)$ filled gas switch that charges a water pulse-forming line in $\sim 150$-ns. Water transmission lines transport energy via self-closing water switches to a water-vacuum interface that is generally a source of high inductance compared to a system that did not require such an interface. The parallel modules are typically joined on the water side of a water/ vacuum interface. The energy is transported via magnetically insulated transmission lines to the load, which may be a dynamic materials experiment, or a radiation effects experiment. This architecture has been utilized for decades to generate mega-ampere currents on the submicrosecond time scale. Examples of such drivers at Sandia National Laboratories include PROTO II [4], SATURN [5], PBFA II [6], and $Z[7,8]$.

In the conventional accelerator architecture, the multimegavolt, mega-ampere gas switch historically constrains the reliable operation, performance, and constitutes a significant fraction of the pulsed-power component maintenance of the system. The gas switch must hold off full 
system voltage for $\sim 1.4 \mu \mathrm{s}$, switch with nanosecond precision, and conduct full module current. It is usually the last command-triggered device in the system, which makes it the predominant controlled device that allows the system to have flexibility for various experimental campaigns. It is generally filled with compressed sulfur-hexafluoride gas and triggered via a focused laser beam with a few nanosecond pulse width. There is a compromise between increasing the size of the switch to operate reliably in the megavolt regime (operate at lower electric fields) and minimizing its inductance (length). There is, therefore, inherent tradeoff between providing flexibility in the design of the device for multiple operating points, and having sufficient reliability at the highest possible operating voltage and current of the system.

This manuscript is the second in a series of articles describing the design and development of the lasertriggered gas switch (LTGS) for the refurbished $Z$ accelerator and Sandia National Laboratories. The first article [9] introduced the design requirements, the motivation for the work, and the performance of the original design at a 5.4-MV level on a developmental test module. This manuscript focuses on the scaling of a 5.4-MV laser-triggered, $\mathrm{SF}_{6}$ filled gas switch to a reliable operating level of 6.1MV for utilization on the recently refurbished $Z$ pulsedpower driver depicted in Fig. 1 [8].

The required operating level for the LTGS increased $25 \%$ over the $6 \mathrm{yr}$ accelerator design effort with no allowable increase in the physical footprint or inductance of the device. Initial design studies indicated that a total peak current of 26-MA into a baseline load (the baseline load is defined as a 20 -mm radius, 20-mm long single wire array) could be achieved with each of 36 gas switches operating at

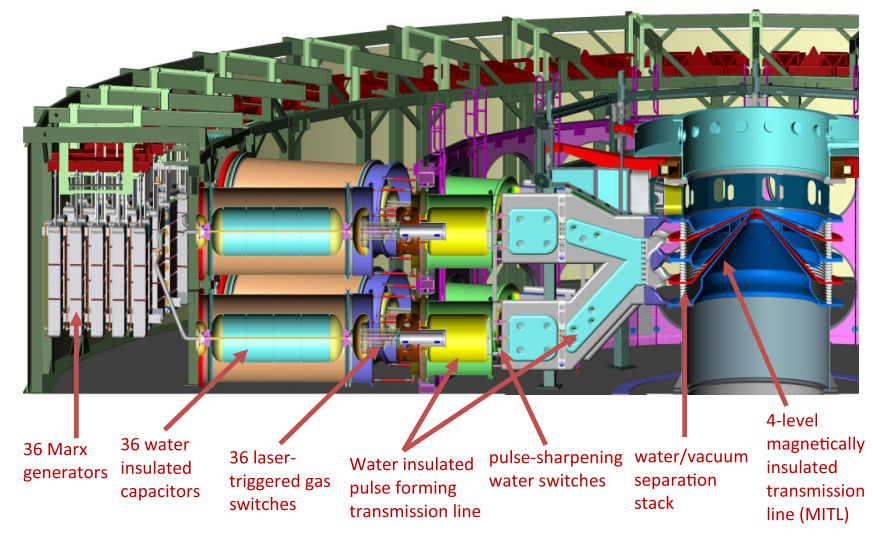

FIG. 1. (Color) Cross section of the refurbished $Z$ at Sandia National Laboratories [8]. The 36 Marx generators, 36 water insulated intermediate storage capacitors, and 36 laser-triggered gas switches are submerged in mineral oil for electrical insulation. The pulse-forming lines and pulse-sharpening switches are submerged in deionized and degassed water. A multilevel insulator stack separates the water section from the central vacuum region where magnetically insulated transmission lines transmit power to the load. $\sim 5$-MV and 0.60-MA. During the design of the refurbished accelerator, system requirements for the LTGS for reliable 26-MA operation into the baseline load changed. Inefficiencies in the transition between pulse transformers, increases in the inductance of the transition from vertical water transmission lines to horizontal magnetically insulated transmission lines, higher inductance in vacuum resultant from changes in the load position for improved diagnostic access, and conservatism in vacuum power flow requirements meant that, to achieve a load current of 22-MA, each LTGS would need to operate at 5.4-MV and 0.70-MA. In the system as it exists today, to achieve $\sim 25$-MA into the baseline load, the LTGS must operate in excess of 6.1-MV and 0.79-MA.

An overview of the 6.1-MV LTGS is given in Sec. II with details of the challenges that faced the development effort given in Sec. III. A summary of the design modifications for reliable 6.1-MV operation is given in Sec. IV. A switch jitter discussion and future work are summarized in Secs. V and VI, respectively.

\section{THE MULTISTAGE LASER-TRIGGERED GAS SWITCH}

The LTGS designed for the refurbished $Z$ accelerator evolved from the Rimfire gas switch first implemented on Hermes III [10]. The modified LTGS presently used in the refurbished $Z$ is depicted in Fig. 2.

Each LTGS is comprised of two sulfur-hexafluoride $\left(\mathrm{SF}_{6}\right)$ insulated gas switches in series: a laser-triggered portion and a self-closing (cascade) portion. The trigger and cascade section share the same volume of $\mathrm{SF}_{6}$ gas,

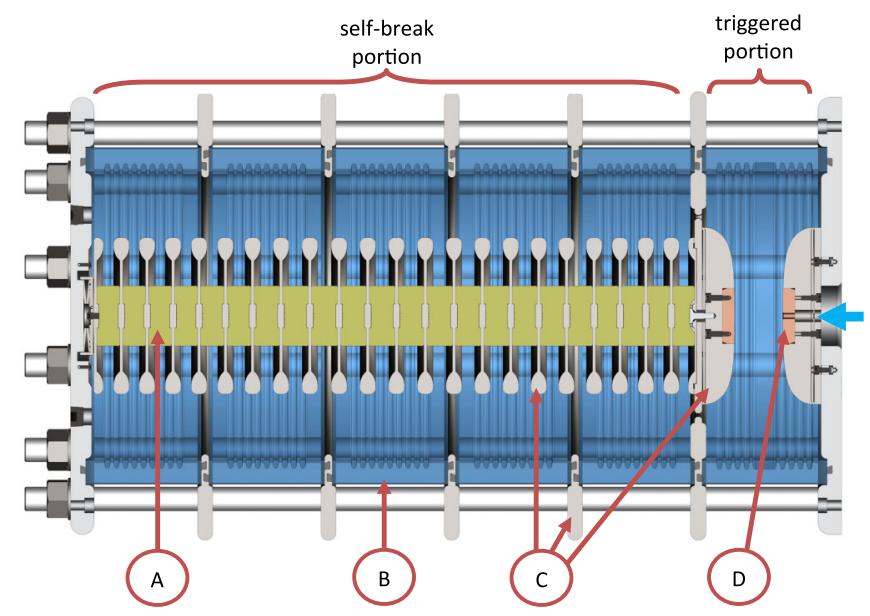

FIG. 2. (Color) Cross section of the 6.1-MV LTGS for the refurbished $Z$ accelerator. The switch is cylindrically symmetric about the laser beam entry path (large blue arrow on the right side). Material: (A) slurry cast polymethyl methacrylate (acrylic), (B) monomer cast acrylic manufactured by C-Lec Plastics, (C) stainless steel 304, (D) 10\% infiltrated copper, 90\% tungsten particulate that is $\sim 3 \mu \mathrm{m}$ in size called Elkonite $50 \mathrm{~W} 3$. 
therefore, the peak intergap electric field in the trigger section and the peak intergap electric field in the cascade section are intentionally matched and conservative enough to optimally compromise achieving an acceptable prefire rate and acceptable jitter. The triggered portion and cascade portion are designed to maintain a uniform peak electric field profile along the desired breakdown axis (at a radius of $\sim 7.5-\mathrm{cm}$ on the electrodes). The insulating gas is contained by a segmented and contoured insulator to maximize the surface tracking length. The $\mathrm{SF}_{6}$ gas is turbulently purged within $\sim 1.5$ seconds after a discharge to ensure a clean environment for the subsequent charge phase.

The voltage impressed across the triggered portion during the charge phase is $\sim 15 \%$ of the total voltage across the switch. The LTGS is triggered by a $\sim 20-\mathrm{mJ}, 266 \mathrm{~nm}$ beam with a 4-ns full width half maximum that enters through the anode electrode and is focused into the center of the 4.6- $\mathrm{cm}$ trigger gap by a $50-\mathrm{cm}$ singlet coated with magnesium fluoride. The laser used for each of the 36 switches, manufactured by New Wave Research, produces $\sim 30-\mathrm{mJ}$ at 266-nm (frequency quadrupled Nd:YAG). The beam is directed into the switch via six mirrors and passes through eight fused silica surfaces (43-mm of glass propagation) for a total path length of $11-\mathrm{m}$. The final focusing optic is a 50-cm singlet coated with magnesium fluoride, an antireflective. The total energy that arrives at the switch with pristine optics is $\sim 20$ - $\mathrm{mJ}$, or $\sim 60 \%$ of the energy at the output of the laser head [11]. The LTGS and its surrounding infrastructure are depicted in Fig. 3. Each switch is equipped with a photomultiplier, encapsulated in an aluminum housing and mounted on the current return structure, which is aimed at the trigger gap. This ensures alignment of the focused laser spark into the triggered gap prior to a shot while the switch is submerged in oil [12].

The fixed focal length lens ionizes a channel of $\mathrm{SF}_{6}$ electrically closing a portion of the trigger gap, which distorts the electric field elsewhere and causes it to close. For the empirically discovered optimum ratio between focal length and gap length of the trigger section $[9,13]$ for 20-mJ laser energy at the switch, the runtime (time between laser spark initiation and electrical closure of the gap) is $\sim 10$-ns with $\sim 1$-ns jitter. The laser, optic path, trigger section gap distance, and electrode geometries used for the 5.4-MV switch are the same as for the 6.1-MV switch.

The cascade section has 22 individual gaps of varying length to maintain a constant peak field between gaps. The axial peak field along the outer edge of the cascade electrode is $208 \pm 6-\mathrm{kV} / \mathrm{cm}$ for $6.1-\mathrm{MV}$ operation. The electric

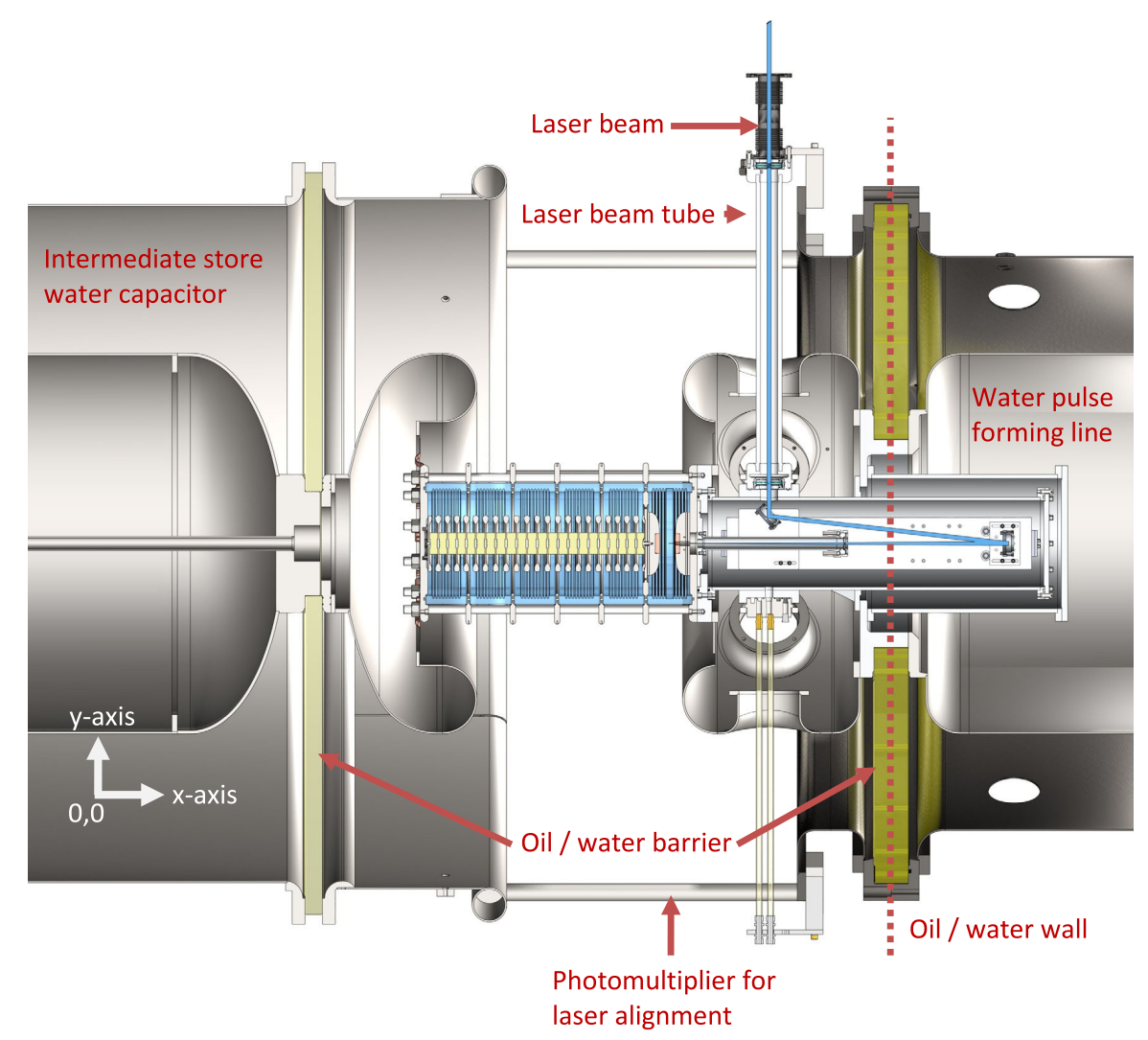

FIG. 3. (Color) Cross section of the intermediate-store water capacitor, the laser-triggered gas switch, the final optics assembly, and first water insulated pulse-forming stage. The gas switch is submerged in mineral oil. The intermediate store is charged negatively in $\sim 1.4-\mu \mathrm{s}$. The laser beam is transported via a Teflon tube and focused by the 50 -cm final focusing lens to the center of the trigger gap. 


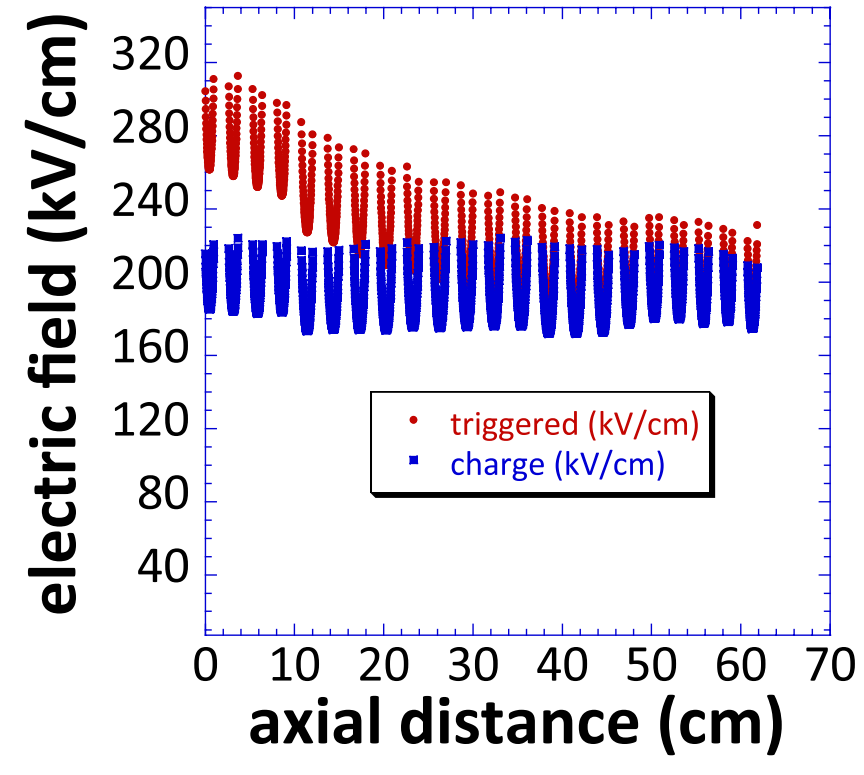

FIG. 4. (Color) Electric field profiles along the axis of electrical closure (radius of $\sim 7.5 \mathrm{~cm}$ ) in the cascade section for the charge and triggered phases.

field across the surface of the insulators that separate the cascade section electrodes is $\sim 98-\mathrm{kV} / \mathrm{cm}$ at $6.1-\mathrm{MV}$, a ratio of $\sim 2.1$ compared to axial peak field. This ratio is much lower than other megavolt $\mathrm{SF}_{6}$ gas switch designs $[14,15]$ making this system very sensitive to material choice and appropriate material handling protocols [9].

When the trigger gap closes, there is an increase in peak electric field of $60 \%$ from $208-\mathrm{kV} / \mathrm{cm}$ to $325-\mathrm{kV} / \mathrm{cm}$ cascade gap adjacent to the trigger section. The capacitive coupling between the trigger section and the cascade electrodes distributes the trigger section voltage over approximately half the cascade gaps. The field profiles for the charging phase and the triggered phase are depicted in Fig. 4.

The cascade section runtime, or time between electrical closure of the trigger section and electrical closure of the cascade section, is $\sim 40$-ns with less than 6-ns jitter, $50 \%$ of which is attributed to the first cascade gap adjacent to the trigger section.

\section{A SINGLE MODULE TEST STAND COMPARED TO THE 36-MODULE REFURBISHED $Z$}

The design effort for the initially deployed LTGS for the refurbished $Z$ was conducted on an engineering module called $Z_{20}$ that replicates one module of the refurbished $Z$ accelerator from the Marx to the interface with the water convolute, with the exception that $Z_{20}$ terminates into an undermatched resistive load [16,17]. Over 3000 shots were taken on $\sim 100$ switch builds on $Z_{20}$ from October 2003 to May 2008.

This test module electrically replicated one module of $Z$ during the gas switch charging phase. A switch design that exhibited a reliable operating point, referred to as the baseline design, was developed at $5.4 \mathrm{MV}$ and $0.70 \mathrm{kA}$ for the initial operation of the refurbished $Z$ [9]. Under these conditions the switch jitter was $\sim 5$-ns, the prefire rate was less than $0.1 \%$, the average lifetime of the switch was greater than 150 shots, and the flashover rate was less than $0.1 \%$.

$Z_{20}$ did not replicate electrical and mechanical conditions after the triggering of the LTGS. Electrical resonance of the system was not replicated because the test module terminates into a matched resistive load rather than into an inductive load as in $Z$, lessening the total coulomb transfer by approximately a factor of 2 . The dynamic mechanical stress due to the interaction of the expanding shock wave produced by 252 water switches in Z's pulse-forming section with large tank structure were not replicated in the test module. The $\mathrm{SF}_{6}$ gas purge system installed on the test module purged switches much faster and with greater turbulence than the system designed for the refurbished $Z$. Finally, since the accelerator was refurbished in place, there was significant construction debris that was not totally mitigated or removed before operation of the system [18]. Each of these factors proved challenging in transferring the stable operating point of the LTGS from $Z_{20}$ to $Z$.

After six months of $Z$ operation, performance of the 36 switches was substantially worse than the measured performance on the test module despite use of the exact switch design (the same electrical and mechanical design requirements were used between the two systems, including materials, and cleaning and handling protocols). The performance difference between the 5.4-MV LTGS tested on $Z_{20}$ (details described by LeChien and colleagues [9]) and the initial deployment of the same design in 36 switches on $Z$ is summarized in the first two columns of Table I. The flashover rate increased nearly an order of magnitude with all flashovers occurring at the bottom of

TABLE I. Comparison between the performance of (i) the 5.4MV laser-triggered gas switch (LTGS) on the engineering test module $Z_{20}$; (ii) the cumulative performance of 5.4-MV LTGS originally deployed on the $Z$ accelerator; and (iii) the 6.1-MV LTGS utilized presently on $Z$.

\begin{tabular}{lccc}
\hline \hline & $\begin{array}{c}5.4-\mathrm{MV} \\
\text { switch } \\
\text { on } Z_{20}\end{array}$ & $\begin{array}{c}\text { 5.4-MV switch } \\
\text { initially } \\
\text { deployed } \\
\text { on } Z\end{array}$ & $\begin{array}{c}\text { 6.1-MV switch } \\
\text { currently } \\
\text { deployed } \\
\text { on } Z\end{array}$ \\
\hline Prefire rate & $\sim 0.1 \%$ & $\sim 3 \%$ & $\sim 0.1 \%$ \\
Total jitter (ns) & 6 & 17 & 6 \\
Trigger section & $\sim 1$ & $\sim 3$ & $\sim 1$ \\
jitter (ns) & & $7 \%$ & $\sim 0.2 \%$ \\
Flashover rate & $<1 \%$ & $\sim 10$ & $>75$ \\
Replacement & $>150$ & & \\
interval (shots) & & & \\
\hline \hline
\end{tabular}


TABLE II. Summary of performance improvements due to design changes when increasing the LTGS operating level from 5.4-MV, 0.7-MA to 6.1-MV, 0.79-MA. The columns represent design iterations and are numbered from left to right as follows: (i) the initially deployed switch on $Z$ after the refurbishment [9], (ii) segmenting the cascade insulator of the switch in column (i) with five equally spaced segments and improving laser alignment in the triggered gap [12], (iii) implementing solid acrylic cascade spacers, scalloping the insulator interior surface, implementing Elkonite 50W3 trigger section electrodes, and a monomer-cast trigger section insulator; and (iv) implementing the fast purge and increasing the mechanical integrity of the cascade section. Column (iv) represents present performance of the LTGS on $Z$.

\begin{tabular}{lcccc}
\hline \hline & Design (i) & Design (ii) & Design (iii) & Design (iv) \\
\hline Prefire rate & $\sim 3 \%$ & $1.5 \%$ & $0.7 \%$ & $\sim 0.1 \%$ \\
Total jitter (ns) & 17 & 6 & 6 & 6 \\
Trigger section & $\sim 3$ & $\sim 1$ & $\sim 1$ & $\sim 1$ \\
$\quad$ jitter (ns) & & & & \\
Flashover rate & $7 \%$ & $1.8 \%$ & $0.5 \%$ & $0.2 \%$ \\
Replacement & 10 & 30 & 50 & $>75$ \\
$\quad$ interval (shots) & & & & \\
\hline \hline
\end{tabular}

the insulator housing, indicating the cause is some contaminant that preferentially locates on the bottom of the switch. Half of all flashovers were prefires (premature electrical switch closure) which can be detrimental to the machine experiment depending on the type. The probability of a flashover occurring within the next two shots of a switch that had flashed on a prior shot is greater than $50 \%$, effectively resulting in the need to replace switches that flashed, but did not prefire. The high prefire rate necessarily increased switch jitter, decreasing the precision of fast-diagnostic synchronization at the load.

Several switch design issues were identified and addressed to improve reliability in the harsher environment of $Z$. There were several modifications to the cascade section insulator to mitigate flashovers including segmenting and scalloping the insulator surface. More robust electrode materials were used in the single channeling trigger section to accommodate higher coulomb transfer. Internal modifications were added to increase impact resilience upon machine discharge (increase lifetime), and an immediate and turbulent gas purge was added to increase reliability of the switch. Modifications in these areas over several rolling upgrades decreased switch jitter from 17 to $6 \mathrm{~ns}$ (factor of 3), decreased random flashover from 7\% to less than $0.2 \%$ (factor of 35 ), decreased the prefire from $3 \%$ to less than $0.1 \%$ (factor of 30 ), and increased the switch replacement interval from $\sim 10$ shots to greater than 80 (factor of 8) at a peak operating level of 6.1 MV. These improvements are summarized in Table II and the details of these modifications are described in the following sections.

\section{MODIFICATIONS TO INCREASE RELIABILITY AT 6.1-MV ON THE REFURBISHED $Z$}

There are four major categories of improvements to switch performance to accommodate 6.1-MV, 0.79-MA operation on $Z$. They are (i) reducing the prefire probability of the cascade insulator, (ii) reducing the probability of prefire caused by severe electrode erosion, (iii) adding a turbulent gas purge to remove contaminants from the switch after a discharge, and (iv) increasing switch lifetime by increasing the mechanical integrity.

These improvements, summarized in Table II, are detailed in the following section. All of the experiments detailed in this section were conducted on $Z$, except where specifically noted. Given the difficulty and expense of dedicated pulsed-power experiments on the $Z$ accelerator, some of the design iterations detailed below were implemented at the same time as others. Therefore, many improvements detailed in Table II are attributable to groups of design changes.

\section{A. Reducing prefire probability of the cascade insulator}

Under pristine operating conditions, a straight (zerodegree) insulator is most resilient to electrical flashover in compressed $\mathrm{SF}_{6}$ gas [19]. Resistance to flashover of an insulating surface is significantly compromised if the surface resistivity decreases due to the presence of a localized surface contaminant, especially one that may result in a concentration of surface charge [20]. In practice, eroded electrode material and metal vapor are deposited on an insulator surface with each discharge, resulting in continuously worsening surface conditions.

After a series of shots, contaminants were collected from the interior surface of the outer housing insulator at various azimuths. Scanning electron microscope images revealed the debris to be stainless-steel spherical particulate $\sim 50 \mu \mathrm{m}$ in diameter, found in substantially greater concentration at the bottom of the housing, most likely originating from ejected stainless-steel 304 material from the cascade section electrodes. Since all observed flashovers occurred at the bottom of the horizontally installed device, the concentration of debris was the likely cause of failures. The comparison of the relative density of particulates was not specifically measured between the 5.4 and 6.1-MV operating modes, but it is assumed that operating at a higher charge transfer level can only increase the concentration of debris.

To mitigate insulator flashover, it is logical to reduce the likelihood of collecting debris in a manner that is conducive to significantly reducing the surface resistivity, and to reduce the likelihood of total switch closure in the event of streamer initiation along the surface. To reduce the probability of housing flashover three modifications were implemented: (i) the monolithic cascade insulator was partitioned into five segments; (ii) the flat insulator surface 
was contoured to increase flashover length and encourage physical debris separation; and, (iii) the insulator acrylic material was changed from a heterogeneous slurry acrylic compound to a homogeneous monomer acrylic resin.

A segmented insulator was implemented in megavolt gas switches for HERMES III [10,21], PBFA I [22], and PBFA II [23-25]. The benefit of segmenting a monolithic insulator for large pulsed-power accelerators is twofold. First, there is reduced likelihood of switch prefire since a localized flash of a single segment is confined by the segmenting metal rings. Second, for any single segment flash that may occur, less insulator damage is likely since a single segment flash will not conduct full device current. This increases the probability of the switch surviving subsequent shots.

The monolithic cascade insulator was partitioned into five equal segments each with approximately $17 \%$ of the total switch voltage. The energy stored between two subsequent rings is $\sim 20 \%$ of a statically charged monolithic insulator that is a factor of 5 longer, therefore the damage incurred in the event of a single segment failure is much less. [In the case of no segments, 100\% of the energy (current) is available to cause insulator surface damage.] The total insulator flashover rate was reduced from $7 \%$ to $1.8 \%$ and the prefire rate from $3 \%$ to $1.5 \%$, as measured over 1100 switch shots (there are 36 switch shots for one $Z$ shot), simply by partitioning the insulator into five segments. Performance improvement of this design is summarized in column (ii) of Table II.

If a single cascade section flashes during the charge phase, a prefire will only be avoided if the resulting disturbance to the electric field at the cascade electrode stack is not significant enough to exceed the breakdown strength of the gas. The ratio of the radii between the inner surface of the housing insulator and the outer edge of the cascade electrode stack is 1.71. Under these conditions a single insulator flashing increases the peak electric field in the cascade section by $18 \%$, increasing the probability of switch prefire an order of magnitude (from $\sim 0.1 \%$ to $2 \%$ as calculated using self-break statistics for the cascade section under normal operating conditions). This is significantly less than an assured prefire due to total flashover of a nonsegmented insulator. Segmenting the insulator also decreases the probability of flashing in late-time (after switch closure) due to oscillating impulses. Increasing the radial distance between the cascade electrode stack and insulator further mitigates this failure mode, and is the principal reason to increase the ratio of the switch housing diameter to electrode diameter.

Segmenting an insulator requires shortening the insulating length because of the added metal segmenting rings. The average field across the total insulator length increases approximately $10 \%$ from 95 to $105-\mathrm{kV} / \mathrm{cm}$ as a result of the addition of the metal rings. However, the detriment of an increase in average field is far outweighed by the benefit

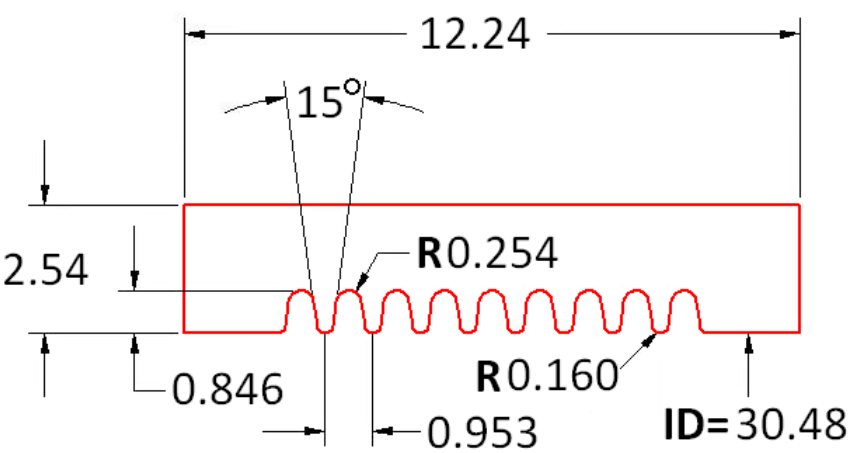

FIG. 5. (Color) Cross section of the single segment of a contoured housing. Dimensions are in centimeters.

of mitigating prefire since the design is conservative from an electrical breakdown perspective.

A scalloped insulator contour was implemented to increase the insulating surface length and inhibit the formation of long chains of debris along the surface. This feature is used in the commercial power industry to increase the flashover strength in debris-contaminated environments compared to a zero-degree insulating surface in $\mathrm{SF}_{6}$ [26] and is beneficial in other debris-contaminated pulse power applications [27]. The shape of the contour used for each segment is shown in Fig. 5. The shape was chosen to maximize the surface length and to maintain an acceptable

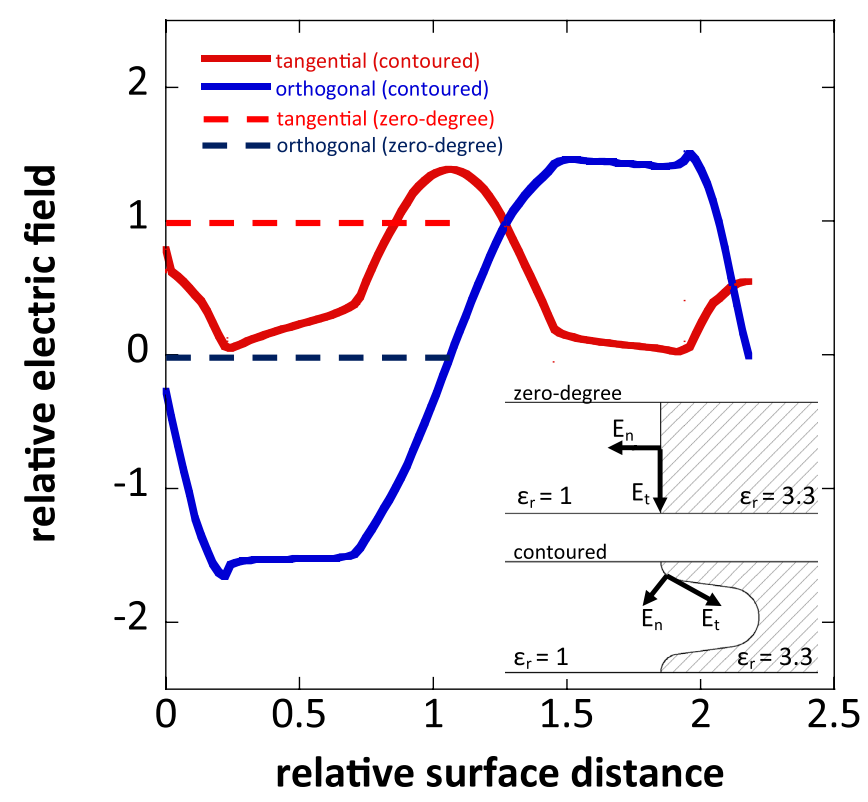

FIG. 6. (Color) Comparison of the 1D calculated tangential and orthogonal components of the electric field along a single groove of the contoured surface profile in Fig. 5 to the tangential and orthogonal field components of a zero-degree insulator of the same axial length. The profiles are for $1-\mathrm{V}$ impressed between the top and bottom surfaces of the legend in the lower right-hand corner. The zero-degree insulator has a surface profile length of 1 and the contoured profile has a surface length of 2.29. 
level of mechanical integrity. This shape increases the insulating surface length by a factor of $\sim 2.29$ compared to a zero-degree insulator.

The contoured shape and dielectric discontinuity (for $\mathrm{SF}_{6}, \varepsilon_{r}=1$ and for acrylic, $\varepsilon_{r}=3.3$ ) creates a lens-like effect reducing the tangential component of the electric field for a majority of the surface profile while increasing the orthogonal component as compared to a flat insulator of the same axial length as depicted in Fig. 6. Though the average electric field must be the same for a contoured insulator as for a zero-degree insulator, it is the tangential field component that will accelerate a streamer along the surface. Therefore, reducing the tangential component over a majority of the surface length at the expense of increasing the orthogonal component reduces the probability of insulator flashover overall.

When implementing a scalloped insulator, the flashover probability was reduced from $1.8 \%$ to $0.5 \%$ and prefire was reduced from $1.5 \%$ to $0.7 \%$, as depicted in column (iii) of Table II, for the same particulate density on the insulating surface.

\section{B. Reducing bulk gas prefire rate in the trigger section}

On the test module $Z_{20}$, the mean time to failure for the baseline switch at an operating point of 5.4-MV and 0.7MA was $\sim 100$ shots. Under these conditions approximately $0.21 \mathrm{C}$ is transferred per shot. This baseline switch used CW70E trigger electrodes (68\% tungsten particulate, $32 \%$ infiltrated copper by weight manufactured by MiTech Metals, Inc.). When the operating point was increased to 6.1-MV and $\sim 0.79-\mathrm{MA}$, the baseline switch failed because of a trigger section prefire at shot 67 . Trigger section self-break shots were conducted to objectively evaluate the performance of the eroded CW70E electrodes. The trigger section was isolated from the cascade section by adding hardware to electrically short the cascade section. The hardware is added in such a way so as to not disturb the electric field profile that is applied to the trigger section. A section of the 6-MV Marx was bussed out to allow a lower voltage pulse, but with the same rise time, to be applied to only the trigger section. The laser was also removed from experiments.

Figure 7 compares the self-break performance of only the gap that is designed to be laser triggered after a series of shots that were conducted under normal operating mode. They three cases are: (i) trigger section self-break shots with CW70E trigger electrodes after 100 shots at 5.4-MV under normal operating conditions; (ii) trigger section selfbreak shots with CW70E trigger electrodes after 67 shots at 6.1-MV under normal operating conditions; and (iii) trigger section self-break shots with Elkonite 50W3 trigger electrodes after 106 shots at 6.1-MV under normal operating conditions. (Elkonite 50W3 is a $90 \%$ tungsten particulate, $10 \%$ infiltrated copper by weight manufactured by CMW, Inc.) From the self-break shots for case (i), the calculated

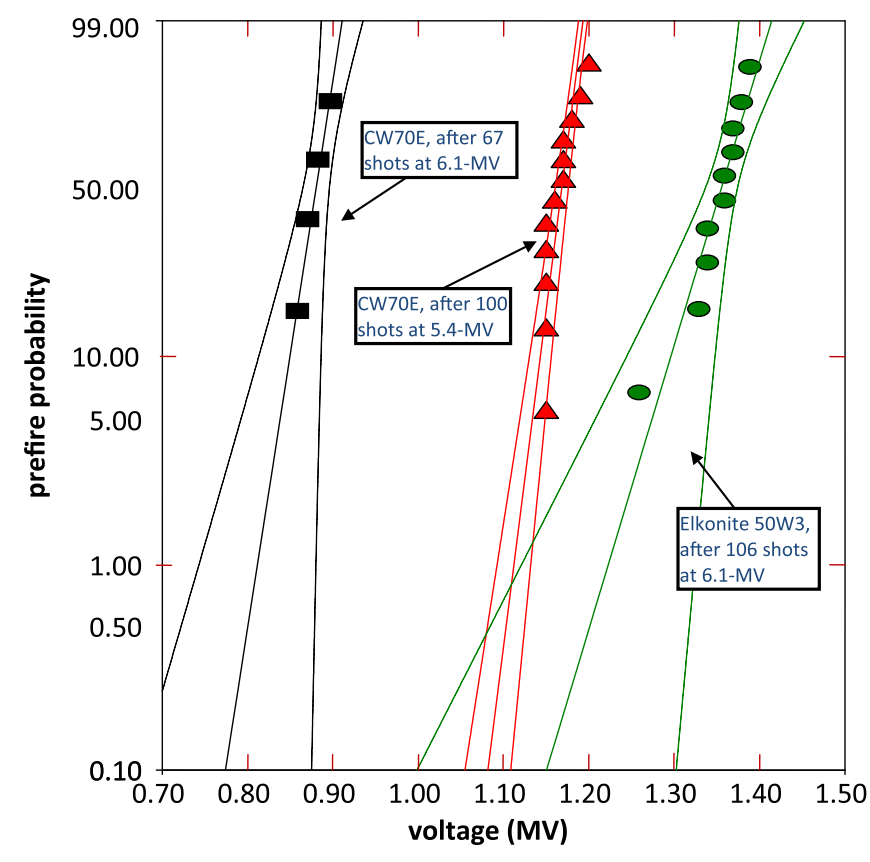

FIG. 7. (Color) Self-break voltage curves for only the gap designed to be triggered by the laser at 48-psia for materials CW70E and Elkonite 50W3. This gap accounts for $\sim 15 \%$ of the total switch voltage when operated under normal operating conditions. The cascade section was electrically shorted out of the circuit to collect this self-break information from only the portion of the switch that is normally triggered. The shots prior to these collected data were conducted at full switch operation under normal conditions. At 5.4-MV there is $\sim 0.21-\mathrm{C}$ transferred per shot, $\sim 0.24-\mathrm{C}$ is transferred per shot at 6.1-MV. The figure is depicted with $90 \%$ confidence bands. The experiments were conducted on $Z_{20}$.

theoretical operating voltage for the trigger section is $\sim 1.07$-MV for a $0.1 \%$ prefire rate, implying a total switch operating voltage of $\sim 7.1$-MV (the trigger section accounts for $\sim 15 \%$ of the total switch voltage under normal operating conditions). This would seem to indicate CW70E electrodes are sufficient for 6.1-MV operation, but the coulomb transfer for case (i) is lower per shot than in reality (coulomb transfer at 5.4-MV versus 6.1MV) so it is misleading to conclude this from only these data. Therefore, another test was conducted to address this [case (ii)]. For case (ii), the calculated theoretical operating voltage for the trigger sections is $0.77-\mathrm{MV}$ for a $0.1 \%$ prefire rate, implying a total switch operating voltage of -5.1-MV (not sufficient for a desired 6.1-MV operating point). The measured failure rate for this case was $1.5 \%$ (1 failure in 67 shots).

The fractional content of tungsten was increased for case (iii) from $68 \%$ to $90 \%$, by weight. For the self-break shots conducted for case (iii), the calculated theoretical operating voltage for the trigger section is 1.15 -MV for a $0.1 \%$ prefire rate, implying a total switch operating voltage of $\sim 7.6-\mathrm{MV}$. The average breakdown voltage for case (iii) is 
49\% higher than in case (ii) (CW70E after 67 shots at 6.1MV). Similar performance was observed when using a similar material constructed of $90 \%$ tungsten particulate, $10 \%$ infiltrated copper manufactured by ATI Wah Chang, a supplier of specialty metal alloys. Other materials were tested during the first phase of LTGS development described in [9].

Comparing scanning electron microscope images, Fig. 8 depicts apparent deep fracturing for the 50W3 material after 106 shots at the 6.1-MV level [case (iii)], but with the copper matrix remaining intact. The CW70E material exhibits deep voids and large locations of liberated copper material after only 67 shots at the 6.1-MV level [case (ii)].

The heterogeneous, slurry-cast, acrylic trigger section insulator was replaced with a monomer-cast acrylic resin material manufactured by C-Lec Plastics, Inc. at the same time the Elkonite 50W3 material was implemented for the trigger section electrodes [summarized in the overall performance improvements between column (ii) and column (iii) in Table II]. Monomer-cast acrylic has been observed to have a hold-off strength as much as a factor of 2 better than slurry-cast acrylic [14]. Though it is more expensive than slurry-cast acrylic, frequent gas switch refurbishment
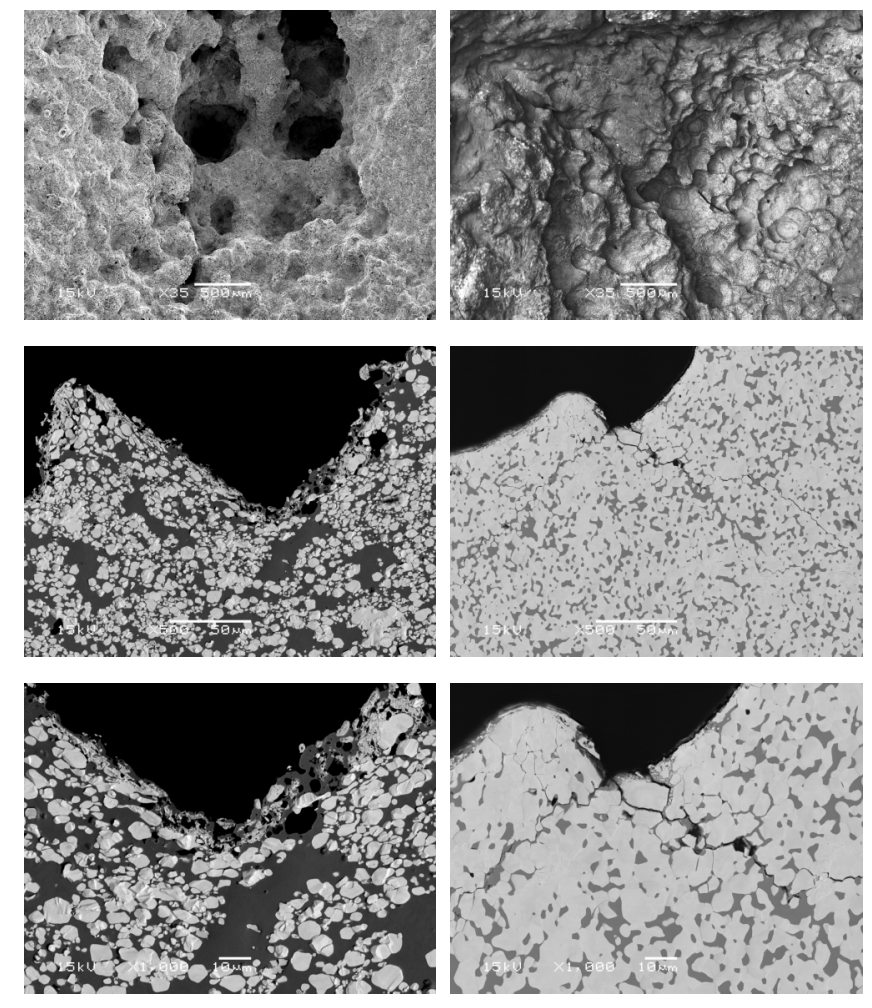

FIG. 8. Backscattered electron micrograph for CW70E (left) after 67 shots at 6.1-MV and for 50W3 (right) after 106 shots at 6.1-MV. The top picture for each material is a top surface view of an arc region. The middle and bottom pictures are cross sections of the bulk material through an arced region at two different scale factors. is considerably more expensive than raw material costs. Monomer-cast insulators are presently used for the trigger housing and the cascade insulator housings will be replaced shortly.

\section{Implementing a fast and turbulent purge with contaminant free $\mathrm{SF}_{6}$}

During the development of the 5.4-MV switch on $Z_{20}$ it was discovered that controlling the dew point and oil concentration level of the $\mathrm{SF}_{6}$ gas, in addition to minimizing the time between a shot and the expulsion of gas from the switch, were critical for reliable switch performance. No experiments were specifically conducted to quantify the effects of water or oil concentration, or to optimize the purge rate, but rather limits for acceptable operation were established. If the dew point exceeded $-30^{\circ} \mathrm{C}$ at 4.14 bar (60 psia), an order of magnitude increase in the flashover rate of the LTGS and a decrease in the reliability of other system components (Marx and Marx trigger system) seemed to happen at the same time. When the oil concentration in the gas exceeded $\sim 25 \mathrm{ppm}$, switch jitter increased by an order of magnitude seemingly due to the inability of laser to acceptably close the trigger gap despite perfect alignment. The purge rate was maximized to remove metal vapor and other by-products as turbulently as possible after a shot as described in [9].

The original $\mathrm{SF}_{6}$ supply system for the refurbished $Z$ did not specifically consider each of these items. Defining and implementing these requirements became one of the most significant system modifications leading to reliable gas switch performance at the 6.1-MV level. On the initial $Z$ system at 3.17-bar (46-psia), a switch-volume (50-L) was purged in $\sim 30$-s; two switch volumes purged in 80 -s. In the improved system, centrally controlled valves for each switch purge a switch volume in less than $7 \mathrm{~s}$ with more than three switch volumes purged in $\sim 60$-s. The delay from an electrical discharge within the switch to the start of a purge cycle decreased from 5 -s to $\sim 1.5$-s with the improved system. The system is presently configured for the most rapid and turbulent purge possible given the fixed infrastructure.

Gas dryers are maintained to ensure the system $\mathrm{SF}_{6}$ has dew point of no more than $-70^{\circ} \mathrm{C}$. All switch gas is passed through oil absorbing pillows, reducing oil contamination to less than $5 \mathrm{ppm}$. The gas is filtered with a $2-\mu \mathrm{m}$ particulate filter before entering the switch.

Column (iv) of Table II summarizes the performance improvements due to implementation of a well controlled gas supply system and improved mechanical integrity of the switch (described in the next section). Though the changes were implemented in parallel, the authors attribute a majority of the reduction in flashover rate from $0.5 \%$ to $0.2 \%$ and the prefire rate from $0.7 \%$ to $0.1 \%$ [column (iv) of Table II] to the implementation of a well controlled gas system. 


\section{Increasing the mechanical integrity of the switch}

The LTGS is rigidly connected to the water pulseforming line through the oil/water barrier at the oil/water wall (see Fig. 3) and cantilevers toward the intermediatestore capacitor on the oil side. It is rigidly coupled to allow a closed line of sight from the laser to the trigger section. The switch is held together in compression by exterior nylon rods, as depicted in Fig. 2. Therefore, the mechanical rigidity is highly reliant on the fabricated tolerance of the housings, electrodes, and spacers.

A dynamic mechanical stress is applied to the water tank structure by the electrical closure of the 252 water switches on a short pulse shot ( $\sim 110$-ns rise time). This stress is applied, in a damped fashion, to the LTGS housing and internals, as well as the optics assembly, through the oil/ water barrier. On a typical $85-\mathrm{kV}$ Marx charge shot ( $\sim 20$-MJ stored), the cantilevered end of the switch accelerates in the $y$ direction (vertical in Fig. 3), peaking at $\sim 157 \mathrm{~m} / \mathrm{s}^{2}(16 \mathrm{~g})$ and oscillating at $16 \mathrm{~Hz}$. In the $x$ direction (horizontal in Fig. 3), the switch accelerates to $\sim 294 \mathrm{~m} / \mathrm{s}^{2}(30 \mathrm{~g})$ and oscillates at $6 \mathrm{~Hz}$. The oscillations exponentially decrease in $1 \mathrm{~s}$. This dynamic loading is a factor of at least 2.3 larger than the anticipated acceleration of $69 \mathrm{~m} / \mathrm{s}^{2}$ that was used for the mechanical design of the switch.

Several design features were implemented to withstand this dynamic loading. The minimum value of the compression force preload of the nylon rods exterior to the switch was increased from $\sim 2500 \mathrm{~N}$ (550-lb force) to $4000 \mathrm{~N}$ (900-lb force), a preload that guarantees sufficient compression of the cascade stack given the elastic nature of the nylon tie rods. The cascade insulator spacers were made of solid slurry-cast acrylic (they were hollow in the baseline design), eliminating the potential flashover mode of the interior surface, and increasing the mechanical integrity of the cascade stack. A feature was added so the movement (elastic compression and expansion) of the cascade stack during dynamic loading prevented the stack from slipping off axis or collapsing completely, since the stack is only held in place by the compression force provided by the nylon tie rods. Increasing the mechanical integrity was a significant factor, though not the most significant, in reducing the prefire rate from $0.7 \%$ to $0.1 \%$ [column (iv) of Table II].

A summary of the four major upgrades and their contributions to switch performance and lifetime improvements is detailed in Table II.

\section{PERFORMANCE ON $Z$}

There are several sources of jitter (variance in the anticipated timing) for arrival of current at the load on $Z$. Timing the arrival of current with fast-gated diagnostics ( $\sim 1$-ns accuracy) is critical to understanding time-lapse information for a given experiment, and in some cases, losing that information can essentially result in a lost experiment. At an individual gas switch failure rate of $\sim 0.1 \%$, the average gas switch jitter is $\sim 6$-ns, providing a total machine load current jitter of $\sim 1$-ns $\left(6 / 36^{0.5}\right)$. Each switch has an individual characteristic runtime that takes several shots to discover when it is installed on the system. After it is discovered, this characteristic runtime is corrected by adjusting the laser arrival time at the switch to synchronize it with the other switches in the system. The LTGS jitter accounts for approximately $66 \%$ of the overall machine jitter after the intermediate-store water capacitors, with the remaining $33 \%$ caused by the untriggered water switches. Reducing LTGS jitter is the focus of present research efforts.

The ratio between the overstress electric field $E_{c}$ in the first cascade gap and the operating pressure $p$ determines the closure time (runtime) and the jitter of the switch upon triggering. A plot of this relationship for several $Z$ shots is shown in Fig. 9. For $E_{c} / p>105-\mathrm{kV} / \mathrm{cm}$ bar, the prefire rate becomes unacceptably high and for $E_{c} / p<$ $95-\mathrm{kV} / \mathrm{cm}$ bar the jitter becomes unacceptably high. This represents a $\pm 5 \%$ window in which switch performance is acceptable. Variance of only a few percent causes a measurable change in an individual switch's characteristic jitter and runtime, and substantially effects overall machine performance.

The ability to have variable pulse shapes resides in the careful control of closure time of the gas switches and the water switch configuration. A comparison of $Z$ magnetically insulated transmission line (MITL) current for a long pulse shot ( $\sim 300$-ns rise time) to that of a short-pulse shot ( 110-ns rise time) is depicted in Fig. 10. Gas switch closure time for each case is also depicted. Ideally, for a short-pulse shot, all gas switches close at the same time for maximum load current and minimal prepulse (current re-

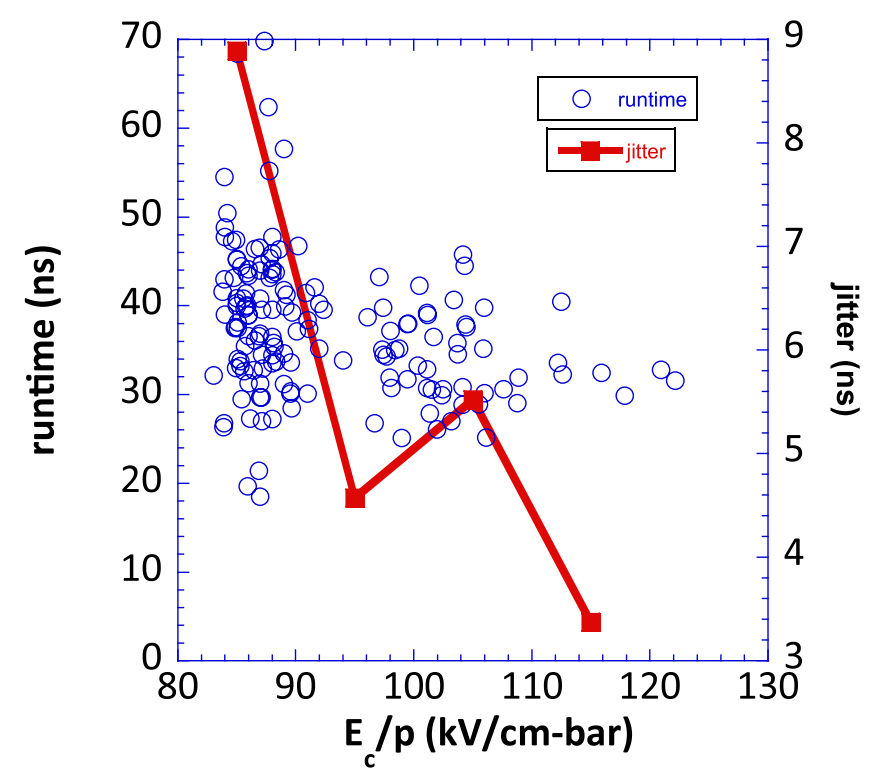

FIG. 9. (Color) Switch runtime and jitter as a function of operating parameters. 


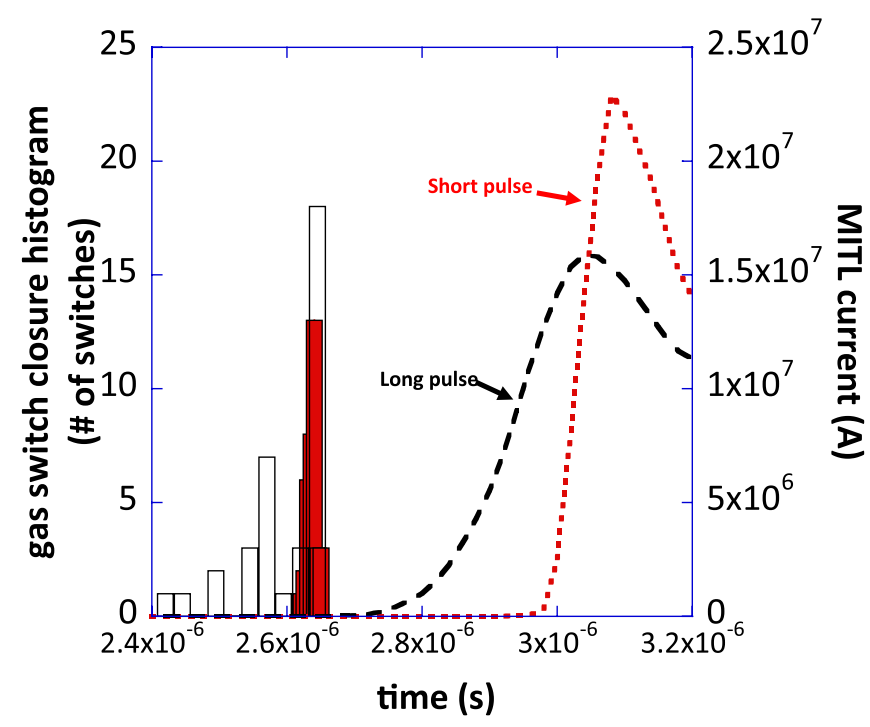

FIG. 10. (Color) MITL current pulse shapes as a function of gas switch closure time.

sulting from displacement current due to capacitive coupling of module elements). For a shaped current pulse, operating pressure is set to maintain a 6-ns individual switch jitter by centrally controlled pressure regulators at each switch and the switches are triggered via the laser and different and controlled times to achieve the desired pulse shape.

\section{SUMMARY}

With over 4000 switch shots to date, and several design improvements, we have developed a 6.1-MV, 0.79-MA LTGS for present $Z$ experiments that is reliable with an acceptable replacement interval. The LTGS initially developed for the refurbished $Z$ accelerator operated at 5.4-MV and 0.7-MA, using SF6 as the fill gas. As the accelerator requirements were increased to provide higher currents to loads of interest, modifications were required to maintain reliability of the LTGS. These modifications decreased switch jitter by a factor of 3 (from 17 to 6-ns), decreased random flashover by a factor of 35 (from $7 \%$ to less than $0.2 \%$ ), decreased the prefire by a factor of 30 (from $3 \%$ to less than $0.1 \%$ ), and increased the switch replacement interval by at least a factor of $\sim 8$ (from $\sim 10$ shots to greater than 75) at a peak operating level of 6.1-MV.

We are pursuing a modification to the most recent design to increase the fraction of voltage in the trigger section, thus increasing overstress wave applied to the cascade section to reduce its jitter. We are also pursuing a new switch design to reduce the switch jitter by an additional factor of 2 while decreasing the random failure rate for an operating level of 6.5-MV and 0.84-MA, peak.

\section{ACKNOWLEDGMENTS}

The authors would like to thank Bruce Tuttle and Jill Wheeler in the Electronic and Nanostructured Materials
Group at Sandia National Laboratories (SNL) for providing dielectric measurements and analysis, Dick Grant in the Materials Characterization Group at SNL for providing SEM images of electrode and insulator materials, and Bob Winters in the Plastics Laboratory at SNL for providing invaluable insulator material preparation services. We thank The Materials Processing and Coatings Laboratory at K-tech, Inc. for providing invaluable electrode material preparation services, and Kiran Androlewicz of K-tech, Inc. for his graphical contributions. The authors extend special thanks to the operations and optics group at the $Z$ accelerator for continuing to provide exceptional operations, service, and maintenance which allow the world class facility to perform experiments on a daily basis.

[1] W. A. Stygar et al., Phys. Rev. ST Accel. Beams 10, 030401 (2007).

[2] W. A. Stygar et al., Phys. Rev. E 72, 026404 (2005).

[3] J.R. Asay and M.D. Knudson, Shock Wave and High Pressure Phenomena (Springer, Berlin, 2005), p. 329.

[4] D. L. Johnson, 1st IEEE International Pulsed Conference, Lubbock, TX (IEEE, Piscataway, NJ, 1976), p. IE2.

[5] D. D. Bloomquist et al., 6th IEEE International Pulsed Power Conference, Arlington, VA (IEEE, Piscataway, NJ, 1987), p. 310.

[6] T.H. Martin et al., IEEE Trans. Nucl. Sci. 28, 3365 (1981).

[7] R. B. Spielman et al., 11th IEEE International Pulsed Power Conference, Baltimore, MD (IEEE, Piscataway, NJ, 1997), p. 709.

[8] M. E. Savage et al., 16th IEEE International Pulsed Power Conference, Albuquerque, NM (IEEE, Piscataway, NJ, 2007), p. 979.

[9] K. R. LeChien et al., Phys. Rev. ST Accel. Beams 11, 060402 (2008).

[10] G. J. Denison et al., 6th IEEE International Pulsed Power Conference, Arlington, VA (Ref. [5]), p. 490.

[11] D. E. Bliss et al., 14th IEEE International Pulsed Power Conference, Dallas, TX (IEEE, Piscataway, NJ, 2003), p. 179.

[12] J.R. Woodworth et al., IEEE 34th International Conference on Plasma Science, Albuquerque, NM (IEEE, Piscataway, NJ, 2007), p. 254.

[13] W. T. Clark et al., 16th IEEE International Pulsed Power Conference, Albuquerque, NM (Ref. [8]), p. 129.

[14] B. N. Turman and D. R. Humphreys, 6th IEEE International Pulsed Power Conference, Arlington, VA (Ref. [5]), p. 347.

[15] M.S. DiCapua, E. K. Freytag, W. R. Dixon, and R. A. Hawley, 6th IEEE International Pulsed Power Conference, Arlington, VA (Ref. [5]), p. 518.

[16] J. P. Corley et al., 14th IEEE International Pulsed Power Conference, Dallas, TX (Ref. [11]), p. 875.

[17] J.R. Woodworth et al., 15th IEEE Pulsed Power Conference, Monterey, CA (IEEE, Piscataway, NJ, 2005), p. 639. 
[18] B.S. Stoltzfus, 17th IEEE International Pulsed Power Conference, Washington, DC (IEEE, Piscataway, NJ, 2009), p. 425.

[19] H. F. A. Verhaart and A. J. L. Verhage, Kema Sci. Tech. Rep 6, 179 (1988).

[20] T. Nitta et al., IEEE Trans. Power Appar. Syst. pas-97, 959 (1978).

[21] G. J. Denison et al., 7th IEEE International Pulsed Power Conference, Monterey, CA (IEEE, Piscataway, NJ, 1989), p. 579.

[22] D. D. Bloomquist, G. R. Montry, S. E. Downie, and G. R. Peterson, 5th IEEE International Pulsed Power Conference, Arlington, VA (IEEE, Piscataway, NJ, 1985), p. 266.
[23] J.M. Wilson and G.L. Donovan, 6th IEEE International Pulsed Power Conference, Arlington, VA (Ref. [5]), p. 361 .

[24] B. N. Turman et al., 4th IEEE International Pulsed Power Conference, Albuquerque, NM (IEEE, Piscataway, NJ, 1983), p. 617.

[25] D. R. Humphreys et al., 5th IEEE International Pulsed Power Conference, Arlington, VA (Ref. [22]), p. 262.

[26] A.H. Cookson, 3rd International Conference on Properties and Applications of Dielectric Materials, Tokyo, Japan (IEEE, Piscataway, NJ, 1991), p. 369.

[27] L.X. Schneider and K. Diegert, Sandia National Laboratories Internal Report, Albuquerque, NM (Sandia National Laboratories, Albuquerque, NM, 1990). 